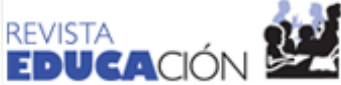

Revista Educación

ISSN: 0379-7082

ISSN: 2215-2644

revedu@gmail.com

Universidad de Costa Rica

Costa Rica

\section{Las interacciones comunicativas en los procesos de enseñanza y aprendizaje en la clase de Estudios Sociales}

García-Calderón, Keiner; Barrientos-Córdoba, Adrián Eliécer; Córdoba-Alfaro, Claudio Israel

Las interacciones comunicativas en los procesos de enseñanza y aprendizaje en la clase de Estudios Sociales

Revista Educación, vol. 46, núm. 1, 2022

Universidad de Costa Rica, Costa Rica

Disponible en: https://www.redalyc.org/articulo.oa?id=44068165039

DOl: https://doi.org/10.15517/revedu.v46i1.47660

\section{(c) $(1) \Theta$}

Esta obra está bajo una Licencia Creative Commons Atribución-NoComercial-SinDerivar 3.0 Internacional. 
Las interacciones comunicativas en los procesos de enseñanza y aprendizaje en la clase de Estudios Sociales

\author{
Interactive Communication Used in Teaching and Learning in Social Studies Classes \\ Keiner García-Calderón \\ Colegio Victoria, Costa Rica \\ garciacalderonkeiner@gmail.com \\ DOI: https://doi.org/10.15517/revedu.v46i1.47660 \\ Redalyc: https://www.redalyc.org/articulo.oa? \\ (DD https://orcid.org/0000-0001-9412-0094 \\ $\mathrm{id}=44068165039$
}

Adrián Eliécer Barrientos-Córdoba

Colegio Los Ángeles School, Costa Rica

abarrientos9002@gmail.com

(D) https://orcid.org/0000-0002-5711-1313

Claudio Israel Córdoba-Alfaro

Academia Teocali, Costa Rica

claudio.cica@hotmail.com

(iD https://orcid.org/0000-0003-3057-1747

Recepción: 12 Julio 2021

Aprobación: 29 Agosto 2021

\title{
Resumen:
}

Este artículo parte de la tesis de licenciatura La clase de Estudios Sociales: Un espacio de interacciones comunicativas en los procesos de enseñanza y aprendizaje.La presente investigación tiene como objetivo analizar las interacciones comunicativas que se desarrollan en la clase de Estudios Sociales y su implicación en los procesos de enseñanza y aprendizaje. Es un estudio cualitativo, con un método fenomenológico, mediante el cual fue posible estudiar la realidad contextual que comparten la persona docente y 27 estudiantes en las clases observadas en un colegio urbano. Las técnicas utilizadas fueron la observación fenomenológica, los grupos de discusión y la entrevista semiestructurada. Se utilizó la triangulación como método de análisis. Los resultados de la investigación son presentados a partir de categorías de análisis centradas en los elementos comunicativos, las relaciones académico personales, y el espacio y organización de aula, así como la mediación didáctica en la asignatura. De estas deriva la construcción de la categoría central empatías o distanciamientos de las interacciones comunicativas en el espacio de clase, como aporte teórico a la temática de estudio. Por último, a partir del análisis de los resultados, se reconoce que la interacción comunicativa influye en cómo cada participante del proceso educativo aprende y se relacionan con la temática de estudio a partir de sus experiencias y el contexto en que se encuentran. Asimismo, se evidencia que la configuración del espacio determina el tipo de interacción y, a su vez, los procesos de enseñanza y aprendizaje.

Palabras CLAVE: Estudios Sociales, Interacciones comunicativas, Contexto, Enseñanza y aprendizaje.

\section{Abstract:}

This article is based on an undergraduate thesis ("Social Studies Classes: An Opportunity for Interactive Communication in the Teaching/Learning Process"). The different communication interactions during a Social Studies class are examined as well as how they impact the teaching/learning process serve as the main objective of this qualitative study. The methodology used is phenomena-based methodology and looks at the contextual setting provided by a teacher and twenty-seven students in an actual classroom in an urban high school. Techniques used for the study include phenomenon-based observation, discussion groups and semi-structured interviews. Triangulation was used as an analysis method. Research are classified based on elements used for communication, personal-academic relationships, the actual space of the classroom and its distribution as well as the didactic aspect of teaching Social Studies as a subject. The aforementioned aspects are based on the construction of a central category called, "Empathy or Distancing in Classroom Interactions used in Classrooms" as theoretical support for the subject of study. Observation and feedback from the study participants allow us to conclude that communication interaction influences how students acquire knowledge and relate to the subject matter studied by using their own personal experience and immediate classroom setting. 
Furthermore, the configuration of the actual physical classroom helps determine the type of interaction that occurs which, in turn, impacts the teaching and learning process.

KeYworDs: Social Studies, Communication Interactions, Context, Teaching and Learning.

\section{INTRODUCCIÓN}

El presente estudio tiene como temática las interacciones comunicativas en educación, con el fin de analizar su influencia en los procesos de enseñanza y aprendizaje que se desarrollan en la clase de Estudios Sociales. La educación es un proceso dinámico que varía según el contexto en el que se desarrolla, por ende, dicho contexto influye en cómo se relacionan las personas y las interacciones que construyen. Por lo anterior, se busca analizar su incidencia en el proceso educativo, enfocado en los de tipo comunicativo y cómo las temáticas de la asignatura forman parte de ello.

En referencia a lo que señala el Programa del Estado de la Nación (2015), en su Quinto Informe del Estado de la Educación, la educación se visualiza como un proceso holístico en el cual se debe tomar en cuenta el entorno en el que sucede el hecho educativo, donde participan y se relacionan estudiantes, docentes, centro educativo, comunidad y familia; agentes que generan interacciones de diverso tipo.

La problemática de la investigación surge a partir de lo planteado en dicho informe, que señala la necesidad de estudiar las interacciones que ocurren en los salones de clase para identificar la influencia que tienen en los procesos de enseñanza y aprendizaje. Este informe señala que el uso del espacio del aula todavía está constituido por una distribución homogénea de estudiantes. Asimismo, los procesos educativos se caracterizan por ser unidireccionales entre el personal docente y el estudiantado, en donde la interacción entre los ambos actores educativos es vertical y limita el contacto entre sí; por lo que apuesta por una búsqueda de una práctica pedagógica que involucre una interacción alternativa.

$\mathrm{Al}$ respecto, el Programa del Estado de la Nación (2017), en el Sexto Informe del Estado de la Educación, explora las condiciones físicas del espacio de aula, el tiempo de clase, la relación social a partir de las metodologías de clase y el papel del personal docente en las interacciones educativas. Sin embargo, no profundiza en la interacción de los actores educativos, por lo que esto representa un vacío académico por investigar en la educación costarricense.

Bernstein (1998) señala que las relaciones entre docentes y estudiantes son tradicionalmente jerarquizadas, por lo que el abordaje de las interacciones resulta necesario para mejorar el proceso de enseñanza y aprendizaje desarrollado actualmente en las clases.

En la misma línea, el currículo de Estudios Sociales plantea la necesidad de considerar “...las interacciones estudiante-estudiante y docente-estudiante, a partir de la interrelación entre conocimientos previos, experiencias del ser humano y la cultura en relación con el conocimiento disciplinar englobado en el campo de los Estudios Sociales" (Ministerio de Educación Pública [MEP], 2016, p. 40). De acuerdo con la referencia anterior, como parte de la naturaleza de la asignatura y los planteamientos del programa de estudio vigente, resulta imperativo comprender la influencia de las interacciones entre participantes del proceso educativo y el conocimiento. Por ello, la presente investigación aborda la problemática relativa a las interacciones comunicativas que ocurren en la clase de Estudios Sociales y la posible influencia de estas en los procesos de enseñanza y aprendizaje.

De lo anterior surgen las siguientes preguntas generadoras: ¿Cómo se manifiestan las interacciones comunicativas entre docente-estudiantes y entre estudiantes en la clase de Estudios Sociales?, ¿cuáles son las manifestaciones del lenguaje verbal y no verbal en las interacciones comunicativas en la clase de Estudios Sociales?, ¿cuál es el trato que se identifica en las interacciones comunicativas que se desarrollan en el aula? y ¿cuál es la incidencia que tienen las interacciones comunicativas en los procesos de enseñanza y aprendizaje en la clase de Estudios Sociales? 
Este artículo está estructurado por los antecedentes basados en estudios previos, con el fin de realizar un acercamiento a la temática en estudio, el referente teórico en que se sustenta la investigación, la metodología empleada para el desarrollo del trabajo en cuanto al tipo y enfoque, los sujetos participantes, las técnicas e instrumentos de los resultados de la investigación que surgen de las categorías de análisis y, por último, las conclusiones basadas en los hallazgos obtenidos.

\section{ANTECEDENTES}

\subsection{Interacciones en prácticas educativas escolares}

El aula es una unidad de análisis metodológica pertinente para estudiar cómo se construyen las interacciones entre docente y estudiantado, y las normas que las podrían regir (Sandoval, 2014). Al introducirse en las dinámicas que se desarrollan en los centros de estudio, Escobar (2015) retoma el lenguaje y la realidad social en los escenarios que contemplan las interacciones entre participantes del proceso educativo en espacios educativos, por lo que realiza una crítica al enfoque tradicional con que se realizan estudios del proceso educativo que se centran en los resultados del éxito académico del estudiantado y no contemplan la incidencia en el proceso de enseñanza y aprendizaje.

\subsection{Interacciones comunicativas áulicas}

En educación, Rizo (2007) plantea que la transmisión de conocimientos, valores y otros elementos se da a través de la interacción comunicativa. En esa línea, Herrero (2012) señala que existen dos tipos de interacción de acuerdo con la planificación o espontaneidad; además, plantea que, para que estas ocurran, se deben considerar las diferentes formas de lenguaje: verbal, no verbal y paraverbal.

Por tanto, el profesorado debe reflexionar sobre el acto educativo y cómo este puede favorecer u obstaculizar las interacciones comunicativas (Keil, 2011). En ese tópico, De Longhi et al. (2012) exponen que en dichas interacciones comunicativas existen roles y, de acuerdo a cómo estos se desarrollen, se configurarán los diversos modelos comunicativos que existen en la clase.

\subsection{Usos del lenguaje}

En el estudio de la interacción didáctica y el lenguaje no verbal, Juárez (2019) indica que, en el proceso comunicativo, el lenguaje no verbal es uno de los que tiene mayor peso, el cual, desde la interacción educativa, debe ser comprendido en relación con la cultura y el contexto social donde se desenvuelven los participantes del proceso educativo. En esa línea, es notable la trascendencia del lenguaje no verbal y la necesidad de considerarlo en el discurso pedagógico como parte del proceso de comunicación, por lo que el personal docente debe considerar esto en su interacción con el estudiantado en la clase (Granja, 2013).

Sumado a lo anterior, Camacaro de Suárez (2008), en sus resultados, plantea que la existencia de la interacción, tanto verbal como no verbal, está mediada por el contexto en el que se envuelven quienes participan del proceso educativo, el cual, a su vez, permite la configuración e intercambio de significados sociales. 


\subsection{Trato entre participantes del proceso educativo}

El trato que se da en los primeros días es fundamental para desarrollar una buena relación entre participantes del proceso educativo. Como lo señala Moya (2010), estas relaciones influyen en los tipos de interacciones que se generan; sobre ese trato, plantea que el personal docente debe tener una apertura con sus estudiantes, pero mantener siempre un límite ético en esa apertura. La autora concluye que, para que exista un buen trato entre docente y estudiantado, debe haber primero una buena comunicación en la clase.

En ese tópico, Keil (2011) encuentra que hay una relación dialógica que se nutre de las emociones generadas entre participantes del proceso educativo, que, a su vez, crean un clima de confianza para el éxito educativo. De igual manera, Chiara (2014) señala que el trato del cuerpo docente hacia el alumnado y entre iguales, influye en el aprendizaje, así como en la actitud hacia este proceso. La calidad de esta relación dependerá en gran medida de las características del personal docente, las cuales repercutirán en el diálogo entre participantes del proceso educativo.

\subsection{Contexto y clima escolar}

Rizo (2007) establece que los actores y actrices educativas, la institución, las interacciones y los procesos educativos que estos generan están influenciados por la cultura y la clase social a la que pertenecen, las experiencias de vida, sus conflictos y las normas que siguen. A su vez, sobre el contexto en el que se desarrollan las interacciones, Pacheco (2013) lo investiga a través de lo que denomina clima escolar, y que este influye en la calidad de las relaciones sociales que se establecen en el aula a través de la percepción del profesorado y el estudiantado, así como de situaciones de aceptación o rechazo que surgen. Por último, Escobar (2015) señala que existen factores externos similares al planteamiento referente al contexto de Rizo (2007), que influyen en los procesos de enseñanza y aprendizaje, de tal forma que sean favorables en la clase.

\section{REFERENTES TEÓRICOS}

\subsection{Interacciones en educación}

El aula es el espacio donde se encuentran inmersas las partes involucradas en la educación, por ende, en ella se generan procesos de interacción, los cuales tienen un vínculo directo con el modelo educativo que se desarrolle. Coll y Sánchez (2008) establecen que el modelo educativo ha sufrido cambios, y que estas rupturas permiten el abordaje de este tema con base en tres principios fundamentales,

...el primero tiene que ver con la crisis del modelo que establece una relación epistemológica jerárquica y unidireccional entre investigación académica y práctica profesional. El segundo, con la aceptación creciente de los enfoques socioculturales y situados de la cognición, el aprendizaje y la enseñanza. Y el tercero, con la importancia creciente otorgada al contexto del aula. (Coll y Sánchez, 2008, p. 15)

En referencia a estos tres principios fundamentales, en el plano educativo, tanto Rizo (2007) como Herrero (2012) señalan que la construcción de los procesos de socialización entre docente-estudiante y entre estudiante-estudiante, se forman a partir del contexto en el que se desarrollan las interacciones de actores educativos, para ello, la comunicación es la base para que se generen estas interacciones, ya sean verticales u horizontales.

Por lo tanto, las interacciones deben ser consideradas en la planificación del personal docente, pues “...no basta con colocar unos alumnos al lado de otros y permitirles que interactúen para obtener automáticamente unos efectos favorables. El elemento decisivo no es la cantidad de la interacción, sino su naturaleza" (Coll, 1984, p. 120), es decir, el abordaje de las interacciones se debe pensar en función de su dinámica. 


\subsection{Tipos de interacciones}

Comprendido el contexto en el que se enmarcan estas interacciones, se identifica la existencia de diferentes tipos, las cuales, según Bixio (2002), son: la interacción sociocognitiva (docente-estudiante); la cognitiva (estudiante-contenido) y la contextual (estudiante-estudiante-objeto de conocimiento-docente-contexto).

Estas interacciones permiten comprender distintos procesos educativos que forman parte de la cotidianidad del aula, así como de la interacción entre docente, estudiante, contexto y contenidos disciplinares. En el plano social, la participación de los actores educativos se caracteriza, según Jauregi (2012), en un intercambio verbal y no verbal de acciones en contextos específicos. El diálogo en este proceso educativo se encuentra influido, en gran medida, por el contexto sociocultural, del cual se construyen significados o códigos que permiten el entendimiento mutuo.

\subsection{Comunicación y lenguaje}

La comunicación es un concepto polisémico, el cual contempla contextos y necesidades. Aguado (2004) plantea que es tanto funcional como semántico, puesto que la comunicación se caracteriza por tener dimensiones como las siguientes: relacional (vínculo entre uno o más elementos diferenciados), perceptiva (permite diferenciar lo que se distingue) y de estructura (permite la relación entre participantes que se comunican).

A su vez, se pueden desarrollar modelos comunicativos en educación, tal y como lo plantea Kaplún (2002), los cuales pueden ser de carácter unidireccional o bidireccional. El primero hace referencia a un modelo educativo tradicional caracterizado por un ente emisor (docente) y el otro receptor (estudiantes), lo que genera roles rígidos en el ambiente de clase. El segundo se fundamenta en un modelo educativo alternativo, en donde hay un intercambio constante de los roles comunicativos, de forma que se alternan entes emisores y receptores en los actores educativos, lo que permite generar conocimiento.

De forma similar, Vigotsky (1995) plantea que las capacidades cognitivas de las personas están influenciadas por el lenguaje, por lo que determina el pensamiento y la forma en cómo se adquiere el conocimiento. Por lo tanto, al considerar el lenguaje como la base de los procesos de enseñanza y aprendizaje, resulta trascendental que se fomenten los intercambios comunicativos entre el personal docente y el estudiantado.

En ese sentido, Vigotsky (1991) plantea que el contexto también determina el lenguaje, y que la cultura en la que se encuentra inmersa la persona establecerá los códigos en que se registra dicho lenguaje, por lo que estos códigos contextualizados son parte del análisis en el tema de las interacciones en el aula.

\subsection{Contexto sociocultural}

Como se ha mostrado en el apartado anterior, la comunicación y el lenguaje están ambientados en una cultura y contexto específico. El proceso educativo, según Cerda (2001):

...transcurre en medio de un marco social o interpersonal donde estamos en constante interacción con otras personas desde la infancia hasta la vejez y, en general, la vida humana resulta difícil de concebir fuera del marco de estas relaciones sociales (p. 30).

Por ende, la educación será la encargada de insertar al sujeto en dicho contexto.

En el ámbito educativo, Vigotsky (1991) expone que, en el desarrollo de la niñez a partir de sus experiencias culturales, no solo se adquieren conocimientos, sino que también se aprenden conductas culturales. Por ello, las instituciones educativas se convierten en espacios donde esto ocurre, lo cual influye en la forma en la que se relaciona cada persona con las demás según dichas experiencias. 
Ahora bien, el centro educativo está influenciado por la comunidad en la que está inmerso, por lo que esta y la familia constituyen la base de la socialización de las personas; tal y como plantea Motta (2010), la relación entre estas instituciones sociales le brinda a la institución educativa un rol de liderazgo en la comunidad.

\subsection{Procesos de enseñanza y aprendizaje}

Desde posturas tradicionales, estos procesos han sido concebidos de forma separada, donde se le asigna al rol docente la enseñanza y al del estudiantado el aprendizaje; de tal postura, Bravo y Cáceres (2006) indican que

...en la mayoría de los salones de clase los estudiantes tienen que atender permanentemente al profesor, quién es poseedor de los conocimientos, los transmite como verdades acabadas, dando poco margen para que el estudiante reflexione y llegue a soluciones, de forma independiente (p.1).

En esa línea, Meneses (2007) expresa que "la enseñanza no puede entenderse más que en relación al aprendizaje; y esta realidad relaciona no sólo a los procesos vinculados a enseñar, sino también a aquellos vinculados a aprender" (p. 32); por lo que los procesos de enseñanza y aprendizaje deben ser considerados de forma bidireccional, es decir, que no se encuentren aislados uno de otro, de tal forma que el estudiantado tenga una participación más activa.

A su vez, el intercambio comunicativo entre participantes del proceso educativo trasciende lo puramente académico, puesto que los procesos de enseñanza y aprendizaje tienen “...como propósito esencial favorecer la formación integral de la personalidad del educando, constituyendo una vía principal para la obtención de conocimientos, patrones de conducta, valores, procedimientos y estrategias de aprendizaje" (Campos y Raubel, 2011, p. 2); por lo tanto, en los procesos educativos, es necesario favorecer procesos de enseñanza y aprendizaje que visualicen a las partes involucradas, tanto en el plano académico como social.

\subsection{Tradiciones epistemológicas}

En la línea de los procesos de enseñanza y aprendizaje es trascendental la relación con las tradiciones epistemológicas, las cuales marcan la pauta a seguir para la planificación, en la que se ve reflejado el estilo docente y su mediación didáctica.

En primera instancia, está la tradición positivista, la cual, según Liceras y Romero (2016), se caracteriza por asignar al rol docente una posición preponderante en la clase como fuente de conocimientos válidos y con un monopolio de la participación en esta, mientras que el estudiantado debe asumir un rol únicamente receptivo.

En segunda y tercera instancia, respectivamente, tal y como señalan Benejam y Pagés (1997), en la tradición humanista es necesaria una mediación didáctica que fomente en los grupos de estudiantes el crecimiento académico y humano, al darle al estudiantado mayor protagonismo; y en la tradición crítica se promueve la criticidad de la realidad en la cual está inmiscuido el proceso educativo por parte de los sujetos que forman parte de ella, pero no solo para analizarla, sino para transformarla. Asimismo, busca romper el modelo jerárquico tradicional basado en un sistema de igualdad.

\section{Metodología}

\subsection{Enfoque de la investigación}

La investigación se encuadra en el enfoque cualitativo, que se caracteriza por "...comprender e interpretar la realidad social en sus diferentes formas y aspectos. Para ello se basa en técnicas que no implican el análisis 
por variables, sino por casos..." (Alesina et al., 1999, p. 77). Por lo tanto, la investigación se desarrolló en un contexto espacial y poblacional específico, en el cual se analizaron las interacciones comunicativas presentes en las clases de Estudios Sociales.

En la misma línea, McMillan y Schumacher (2005) exponen que la investigación cualitativa permite tanto analizar como describir conductas de los sujetos en los planos sociales e individuales, con miras a comprender los fenómenos sociales desde la visión de las personas participantes, por ello, el análisis propuesto parte de la visión de este enfoque.

\subsection{Método de la investigación}

El método desde el cual está orientada la investigación es el fenomenológico de Husserl (1949), en el que se parte de la experiencia de las personas participantes para generar un conocimiento general base. A partir de estas vivencias, se busca describir posibles interpretaciones que permitan crear una noción científica fundamentada.

El método fenomenológico aplicado en educación, según Jaramillo y Aguirre (2012), permite indagar de forma acentuada en las realidades escolares, puesto que explora el estudio de la cotidianidad en la que se encuentran inmersos los actores educativos a partir de un contexto en común.

\subsection{Unidades de análisis}

\subsubsection{Criterios de selección}

Los criterios considerados para la realización de la investigación fueron:

\subsubsection{El centro educativo}

En la selección de la institución educativa se planteó que fuese un colegio público de la Gran Área Metropolitana de Costa Rica, de modalidad académico-diurno, pues son las instituciones que albergan a la mayor cantidad de población estudiantil, con un total de 693 , lo que representa un $71 \%$ del país (Dirección de Planificación Institucional, 2016). Ello amplía el margen de que el estudiantado provenga de diversos contextos socioculturales, elemento primordial para el análisis, dado que ese origen diversifica las interacciones comunicativas investigadas.

Según Rizo (2007), los sujetos están adscritos a un sentir desarrollado en su comunidad, por lo que su interacción estará influenciada por su lugar de procedencia, tanto a nivel cultural como social. Por ende, se buscaron varios colegios que cumplieran con esas condiciones, y resultaron cuatro centros educativos que cumplían con lo establecido, en donde se seleccionó un colegio de Mata Redonda, circuito 1 de la Dirección Regional San José Oeste. Para hacer una evaluación de la aplicación de las técnicas, se realizó un plan piloto en la segunda institución que cumplía con los criterios establecidos.

\subsubsection{Participantes / actantes}

Docente: Para la selección de la persona docente, se determinaron los siguientes requisitos: en primer lugar, laburar en un colegio público académico que sea urbano y diurno a tiempo completo, dada la cotidianidad de las interacciones que tiene con el estudiantado. En segundo lugar, poseer experiencia laboral de al menos cinco años de forma ininterrumpida, con la intención de que tenga un amplio bagaje en interacciones 
comunicativas en el espacio de aula. La selección de la persona docente fue a cargo de la dirección de la institución a partir de los criterios establecidos, con el fin de que el equipo investigador no influyera, lo que garantizó la selección aleatoria de la persona docente.

Estudiantes: En cuanto a la selección de estas personas participantes, se determinó que fuesen de educación diversificada, específicamente de décimo año en el desarrollo del segundo y tercer trimestre, por dos razones; en primer lugar, porque han concluido distintas etapas educativas a través de experiencias entre estudiantes con el cuerpo docente, y, en segundo lugar, porque en undécimo año el ciclo lectivo concluye de manera anticipada. Con respecto a estas características, el total de estudiantes que formaron parte del estudio estaba compuesto de 27 personas. A su vez,

A las personas participantes en el proceso de recopilación de los datos se les facilitó e informó sobre: a) el objetivo de la investigación, así como de las generalidades de ésta, b) beneficios y riesgos de los datos aportados durante la entrevista al ser puestos a análisis, c) la garantía de confidencialidad en el estudio, y d) la información de los resultados obtenidos y analizados, para comprobar la veracidad del aporte que la persona realizó a la investigación. (Barrientos et al., 2018, pp. 67-68).

\subsection{Técnicas de recolección (instrumentos)}

Dada la naturaleza metodológica de la investigación, se utilizaron diversas técnicas para responder las primeras tres preguntas planteadas, las cuales se explican a continuación.

En primer lugar, para la observación fenomenológica se elaboró un instrumento que surgió a partir de los elementos que componen los antecedentes que permitieron seleccionar elementos a observar en el plan piloto. Posteriormente, se incluyeron nuevos elementos a observar en el instrumento oficial que permitiera identificar elementos que influyen en la dinámica de clase en relación con las preguntas de investigación. Esta técnica se aplicó a las catorce lecciones observadas.

En segundo lugar, se encuentran los grupos de discusión, mediante los cuales se elaboraron una serie de preguntas generadoras a partir de la ejecución del plan piloto, los ejes del apartado de antecedentes, así como de las preguntas de investigación; para la aplicación de la técnica se trabajó con grupos de cuatro a cinco estudiantes máximo y se desarrollaron cuatro grupos de discusión.

En tercer lugar, se aplicó la entrevista semiestructurada para la cual se construyó un instrumento específico para la persona docente, el cual contenía una serie de preguntas generadoras sobre los ejes en estudio, a partir de la aplicación del plan piloto, así como de los ejes de los antecedentes. Además, la cuarta pregunta de investigación se resolvió a través de la triangulación como método de análisis.

\subsection{Procesamiento de análisis}

El proceso de análisis de la información inició con la construcción de matrices de clasificación estructuradas que se derivan de la aplicación del plan piloto, los ejes teóricos-temáticos y de la aplicación de las técnicas de investigación, lo que permitió, a través de la triangulación, la creación de categorías de análisis. Estas son: elementos comunicativos de la clase, relaciones académico-personales en la clase, espacio, movilidad y organización del aula, la mediación didáctica docente y la interacción con los contenidos de Estudios Sociales.

Con el objetivo de dar respaldo a los argumentos planteados en el análisis de la información, que permitan un proceso de verificación en la credibilidad de los hallazgos, se implementaron dos tipos de triangulación: en primer lugar, se utilizó la triangulación de técnicas de investigación, la cual consistió en contrastar la información recabada; en segundo lugar, se realizó la triangulación teórica, donde se vinculó la información recogida con las técnicas y las bases teóricas en las que se fundamenta la investigación. En esa línea, Gurdián 
(2007) señala que la triangulación permite entrelazar la visión y aporte de los sujetos participantes, la teoría existente y el análisis de quienes investigan un argumento central.

\section{Resultados}

\subsection{Elementos comunicativos de la clase}

En esta categoría de análisis se abordaron los siguientes indicadores: en el lenguaje verbal se analizó el vocabulario, el tono de voz y el uso de la palabra. Por su parte, en el lenguaje no verbal se abordaron los gestos y expresiones, miradas y silencios.

En el ámbito educativo, los elementos comunicativos se presentan en el momento en que los actores educativos ingresan al aula, dado que desde ese momento inicia una configuración de significados y códigos propios que permiten enviar y captar los mensajes que se desarrollan en el proceso dialógico de la clase (Keidar, 2005).

Comprendidas la educación y la comunicación en su relación dialéctica, el lenguaje cobra un papel central: sin lenguaje no hay comunicación y, si no hay comunicación, las oportunidades de desarrollar el conocimiento se limitan (Vigotsky, 1995). En esta investigación se atiende a dos formas de lenguaje: el verbal y el no verbal.

En cuanto al lenguaje verbal, en la investigación se analizaron el vocabulario, el tono de voz y el uso de la palabra. El vocabulario identificado fue coloquial, según momentos de la clase, en los procesos comunicativos tanto del personal docente como del estudiantado, lo que posibilitó un ambiente de confianza y comunicación accesible; también se identifican, por parte del personal docente, explicaciones con vocabulario sencillo, lo que permitía una mejor comprensión del mensaje por parte del estudiantado.

En lo referente al tono de voz, la persona docente captaba la atención del estudiantado, y lo aumentaba a la hora de llamar la atención o por influencia de emociones, mientras que el estudiantado utilizaba un tono de voz que aumentaba producto de las empatías o al finalizar la clase. En cuanto al uso de la palabra, debido a las clases magistrales que empleó la persona docente, se reflejó una forma unidireccional en la mayor emisión de mensajes. En el caso del estudiantado, el uso de la palabra se dio principalmente fuera de la planificación didáctica del personal docente, a razón de las relaciones que entablaron esta fue bidireccional, debido a los constantes cambios entre sus roles emisores y receptores.

Por su parte, en el lenguaje no verbal, el mismo estudiantado reconoce que es un estímulo visual mediante el cual capta cuándo el docente quiere llamar su atención o cuándo hace énfasis en algo importante. Asimismo, menciona que a partir de las expresiones faciales puede visualizar la cercanía que tanto el docente y el estudiantado puede tener en su proceso comunicativo. Uno de los estudiantes en los grupos de discusión menciona al respecto: “...él siempre está moviendo las manos o cuando va a explicar algo siempre utiliza las manos para hacer énfasis" (Grupo de discusión 1, 21 de agosto de 2017).

En lo que respecta a las miradas, fueron utilizadas como mecanismo de control reconocido por todas las partes que participan de la clase; además, los silencios fueron referidos a momentos incómodos, dado al temor de que la participación genere burla, o que no haya respuesta por parte del alumnado a partir de los planteamientos de la persona docente, así lo expresan las personas estudiantes cuando mencionan: "a veces él explica un tema y, como te dije, lanza una pregunta al aire y tal vez nadie está poniendo atención, entonces se queda ahí esperando" (Grupo de discusión 3, 25 de setiembre de 2017).

En síntesis, se encontró que el lenguaje es la base para el desarrollo de la interacción comunicativa en clase, y que el vocabulario empleado por ambas partes permite la comprensión mutua entre estos; siempre que no se caiga en tecnicismos o una vulgarización del lenguaje que dificulte la comunicación. Por su parte, se evidenció que cuando el modelo comunicativo es bidireccional, el estudiantado tiene un papel más activo 
en la construcción del conocimiento, dado que, si la clase es magistral/unidireccional, el personal docente monopoliza la palabra y se limita la interacción.

En cuanto a lo no verbal, esto tiene gran influencia dado a que, según Moreno (2007), posee un alto porcentaje en lo que se busca transmitir, por lo que debe desarrollarse de forma consciente y acorde con el mensaje, de manera que permita mejorar el hecho educativo. Por último, ambas partes del proceso educativo construyen sus propios códigos comunicativos, lo cual debe tomarse en cuenta dado a que, al desarrollarse una jerga entre estos grupos, permite el entenderse entre sí y mejorar la interacción comunicativa.

\subsection{Relaciones académico-personales en la clase}

En lo referente a esta categoría de análisis, se trabajaron los siguientes indicadores: el trato entre actantes del proceso educativo, la participación, los mecanismos de control y el contexto sociocultural.

$\mathrm{Al}$ ser la educación parte de estructuras sociales, se convierte en un espacio de convivencia en el que "el docente y su grupo de estudiantes participan cotidianamente en los mismos procesos educativos, comparten sentimientos y experiencias de su entorno. Estos aspectos resultan medulares en el desarrollo integral de cada persona y lo preparan para desarrollarse socialmente" (Artavia, 2005, p. 2). Asimismo, los centros educativos tienen una función social (Torres, 1998) en la que el trato entre actantes, la participación que poseen y los mecanismos de control existentes en la clase, se encuentran relacionados al contexto sociocultural.

En la investigación, según los datos de procedencia que brindó la administración del centro educativo, el estudiantado proviene de distintas realidades, tanto por su entorno geográfico como socioeconómico. Según el personal docente entrevistado, esto debe ser tomado en cuenta en el desarrollo de las clases, ya que es posible que, si se parte de la cotidianidad estudiantil, se promueva mayor interés de estos grupos hacia los contenidos de la asignatura. La persona docente en la entrevista señala lo siguiente:

...les digo que levanten la mano quienes de sus papás son profesionales entonces son muy pocas manos las que levantan...hay unos que fueron profesionales y otros que no, en qué trabajan sus papás, trabajan en una fábrica y el mío es contador y el mío es tal cosa entonces ahí es donde trato de ver si les interesa, usted le cambia un poco la dinámica de clase. (Docente, 23 de noviembre de 2017).

$\mathrm{Al}$ respecto, Bedmar (2009) plantea que si el centro educativo se cierra al contexto social, no se podrán construir aprendizajes funcionales, puesto que no se logran vincular los contenidos de la asignatura con la cotidianidad del estudiantado.

El trato en el proceso comunicativo y de socialización parte de las relaciones humanas, las cuales establecen vínculos entre quienes se comunican, por lo que dependerá de su tipo, la relación y el trato que se pueda generar. Además, el tiempo de convivencia es otro de los elementos que interviene en la construcción de un ambiente con un trato que permita, finalmente, los procesos de interacción comunicativa. Por lo tanto, en el ámbito educativo, la medida en que avance dicha convivencia puede ir entretejiendo, entre los grupos involucrados, vínculos de empatía que posibiliten construir un ambiente óptimo para generar un proceso de interacción comunicativa en torno a los procesos de enseñanza y aprendizaje.

Según Sime (2006), las relaciones entre participantes del proceso educativo se establecen en un tiempo y espacio particular, lo que produce la generación de vínculos positivos o negativos que influyen en las dinámicas y en el ambiente de la clase. En el caso de estudio, con el avance paulatino de las clases se fue estableciendo una mayor confianza y socialización; lo cual permitió observar que entre las partes educativas se fue creando una relación o trato caracterizado por ser amigable y flexible, y se promovían espacios de confianza y cercanía en el abordaje, tanto de cuestiones académicas como personales.

Ahora bien, el trato del docente con la totalidad del estudiantado no es el mismo, dado que se puede presentar un trato igualitario o diferenciado. En un plano ideal, este debería ser igualitario con todo el grupo, sin embargo, resulta difícil que cada docente pueda establecer una relación de empatía con todo el estudiantado, empero, esto no exonera al profesorado de propiciar, en la medida de lo posible, una relación y 
trato igualitario. En cuanto a lo académico, Moya (2010) expresa que debe haber una buena relación docenteestudiante si se quiere tener una relación eficaz y didáctica de calidad, pues sin el componente humano se reduce la idoneidad en la enseñanza y el aprendizaje.

El personal docente de este estudio, al ser cuestionado sobre este tema, reconoce que debido a la empatía de unas personas y con otras, el trato es diferenciado, es decir, tiene a algunas personas estudiantes preferidas, esto se muestra al expresar: “...uno tiene empatía, hay otros que no, eso está mucho en la confianza que a uno le den...si hay estudiantes que son preferidos y hay otros que no lo son, pero uno los trata igual..." (Docente, 23 de noviembre de 2017). Lo anterior repercute en que, en caso de existir empatía, al momento en que se genere la interacción, esta permite facilitar la comunicación; por el contrario, si existiese apatía podría limitar o dificultar el proceso comunicativo.

En lo referente a la participación, el personal docente entrevistado reconoce que no considera esto como parte de su planificación didáctica. Se observaron dificultades para generar el diálogo con sus estudiantes, debido a que desarrolla principalmente clases magistrales, las cuales limitan la participación estudiantil, como ejemplo de lo anterior se indica lo siguiente:

...por lo general son clases magistrales, suena feo, pero sí, son clases magistrales, eh, a veces empezamos tratando de generar el diálogo, pero... sí cuesta, sí cuesta; o tal vez a veces estamos hablando...bueno ahora que estuvimos viendo demografía, les da por preguntar muchas cosas, esos temas les gusta. (Docente, 23 de noviembre de 2017).

Esto es un aspecto a considerar, puesto que, como señalan García et al. (2014), el personal docente como coordinador del grupo debe promover espacios de interacción en la clase que configuren espacios de confianza para la participación del estudiantado.

A su vez, los rasgos de la personalidad del estudiantado afectan su participación en clase, dado que, según lo observado en las clases y sumado al aporte de los grupos de discusión, las condiciones de timidez o extroversión afectan; por lo que, de acuerdo con la investigación realizada, existe una relación entre la forma de ser y el nivel de participación de cada estudiante, por lo que el personal docente debe ser reflexivo sobre este punto en su práctica profesional. En los grupos de discusión una persona estudiante menciona

Depende de la persona, es como personalidad, algunos tienen como vergüenza en la clase, si no participan, porque no participan, es por algo de la persona o porque no hay interés o no quieren, y los que participan más porque son más extrovertidos al rato. (Grupo de discusión 2, 28 de agosto de 2017)

Por otra parte, sobre los mecanismos de control, Mejía y Ávila (2009) plantean que son un recurso que tiene el cuerpo docente y que existen varias formas de emplearlo. En el caso de la persona docente observada destacó la silla de los castigados, nombrada así por ella misma, sumado a las miradas, el decomiso de objetos, las preguntas para regular la participación y, en última instancia, el llamado a los padres y madres de familia.

Ante la concentración de las interacciones comunicativas en una persona, la persona docente decidió movilizar de lugar a una estudiante hacia la silla en mención, lo que alteró el flujo de las redes comunicativas que se presentaban en la clase; esto cobra importancia dado que, en la relación establecida, el personal docente envía un mensaje claro de control autoritario y su rol dentro de la dinámica de clase, lo cual puede generar un ambiente negativo si no hace un buen uso del poder que posee y se malinterpreta por el estudiantado. Camacaro de Suárez (2008) plantea que esto genera un patrón de control en el que el personal docente busca justificar su autoridad. Esta actitud de reafirmar el orden y la autoridad es reconocida por el mismo estudiantado participante del estudio al indicar que la persona docente decomisa objetos, llama a las personas encargadas de familia o hace preguntas de control; por ejemplo, una estudiante menciona en el segundo grupo de discusión: "Eso sí, decomisa cosas como teléfonos, después los devuelve... al final de la clase él los devuelve, él lo quita..." (Grupo de discusión 2, 28 de agosto de 2017).

En esa línea, si bien se busca promover interacciones más horizontales entre docente y estudiantado que no promuevan modelos verticales tradicionales, se debe entender que docentes y estudiantes no estarán al mismo nivel, dado que "los profesores no son iguales a los alumnos por . razones, entre ellas porque la diferencia 
entre ellos los hace ser como están siendo. Si fuesen iguales, uno se convertiría en el otro" (Freire, 2002, p. 112). Empero, se reitera que la persona docente debe promover estructuras más horizontales para promover interacciones comunicativas que brinden más participación al estudiantado.

A manera de síntesis, el contexto sociocultural de los actores educativos es un factor influyente en la construcción de procesos socializadores e interacciones comunicativas en la clase que, de ser tomados en cuenta en la mediación didáctica, propicia que sean más verticales u horizontales. A su vez, en el ámbito educativo es trascendental entablar un trato amigable y de confianza, propiciador de interacciones comunicativas, aunque nunca será una relación igualitaria, debido a que siempre existirán afinidades o distanciamientos entre participantes del proceso educativo.

\subsection{Espacio, movilidad y organización del aula}

En lo referido a esta categoría de análisis se abordaron los siguientes indicadores: condiciones físicas y de organización, sectorización y distribución, grupos y subgrupos, así como la movilidad de las partes educativas involucradas.

En la formación profesional de docentes, es frecuente que se enfoque prioritariamente solo lo disciplinar y se deja de lado el abordar elementos como el espacio y su impacto en la mediación didáctica y las redes de interacciones que en ella se generan. Sobre lo anterior, el Programa del Estado de la Nación (2017), en su Sexto Informe del Estado de la Educación hace hincapié en la importancia de la dimensión espacial y su vínculo con los procesos de enseñanza y aprendizaje.

En ese tópico, el aula debe ser entendida como el espacio físico y los objetos o elementos que la componen o son parte de esta. Ahora bien, estos elementos configuran la forma en cómo se desarrolla la clase, por lo que su planificación puede estimular o limitar la interacción comunicativa que se produce.

En relación con lo anterior, desde la estructura tradicional, el aula se organiza con una disposición en la que los pupitres se acomodan en filas en dirección a la pizarra, esto limita la interacción comunicativa del estudiantado, puesto que su panorama está dirigido hacia esa dirección principalmente. Tal y como indican Campos et al. (1993), en relación con la organización del aula, "es importante que el maestro cambie la distribución de pupitres, pues este cambio en sí puede generar interés en los niños, se rompe la monotonía y se establecen interacciones con diferentes niños del aula" (p. 90).

En el caso en estudio se logró evidenciar este aspecto. Se observó que la persona docente mantuvo la estructura tradicional; empero, en ciertas ocasiones buscó variar la orientación del estudiantado en el espacio del aula. Pero, si el cambio no va acompañado de una planificación didáctica y no tiene un propósito, podría no variar las interacciones comunicativas desarrolladas, tal y como ocurrió en estas clases.

A partir de lo observado se estableció el término de radios de interacción comunicativa, entendido como el alcance inmediato que posee el estudiantado en el espacio para interactuar con sus pares. En una de las observaciones se identificó que mientras una estudiante acapara la interacción con sus pares, otra estudiante, por su ubicación, interactuaba solo con elementos físicos del aula, como se muestra en la Figura 1. 


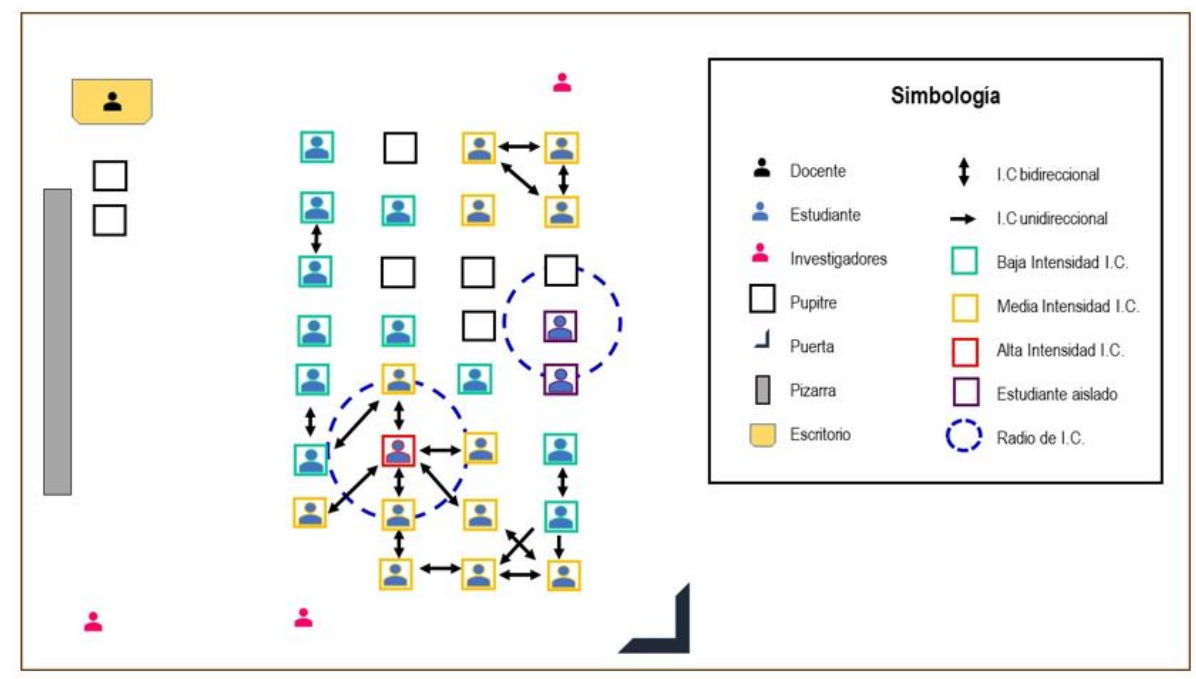

FIGURA 1.

Mapa de los radios de interacción comunicativa.

Fuente: Barrientos et al. (2018).

Mantener esa estructura tradicional, según Torres (1998), replica el sistema del panóptico de Bentham, en el cual el profesorado es el centro desde el cual se controla y observa al estudiantado, por tanto, el estudiantado llega a tener más interacción con los objetos del aula que con las personas que allí se encuentran.

En lo que refiere a la conformación de subgrupos, Guardia de Viggiano (2009) indica que esto se realiza, en gran medida, debido a la existencia de intereses en común o al contexto sociocultural que comparte el estudiantado. De acuerdo con la percepción del estudiantado que formó parte de la investigación, este señala que los subgrupos se forman a partir de intereses y afinidades en común, lo que la persona docente también reconoce, según lo que observa en la clase; sin embargo, a la hora de la conformación para grupos de trabajo, no siempre utiliza este criterio. Ahora bien, según lo que plantea Coll (1984), no basta solo con configurar los pupitres y poner al estudiantado junto para que se genere automáticamente una interacción, sino que la persona docente debe pensar la forma en cómo se va a desarrollar dicha interacción. En una de las clases observadas se ejemplifica este argumento, dado que la persona docente conformó subgrupos a medida que ingresaban a la clase; sumado a esto, la mediación didáctica planteada no era acorde a la actividad desarrollada, por ende, la actividad en subgrupos limitó las interacciones comunicativas.

En consecución con el análisis espacial y su influencia en las interacciones comunicativas, en el trabajo de campo se observó la conformación de los subgrupos en la clase y la forma en la que las personas se encontraban distribuidas. Se estableció el concepto de redes de interacción comunicativa, las cuales visualizaban la frecuencia y dirección de las interacciones, los elementos físicos y su posición en el aula, el modelo de comunicación (unidireccional o bidireccional), así como la movilidad de cada participante del proceso educativo. Lo anterior permitió identificar zonas del aula donde se intensificaron las interacciones comunicativas entre estudiantes, así se estableció el concepto de sectorización. Ambos conceptos surgen a partir de la comparación de las observaciones fenomenológicas de cada persona investigadora.

En la Figura 2 se identifican las redes de interacción comunicativa observadas en una clase, en donde existe un espacio vacío por parte del estudiantado, situación que la persona docente confirma al mencionar: “... siempre dejan sectores vacíos...El centro, el centro siempre lo dejan vacío, siempre se me sientan a los lados, hay unos que son muy pegas" (Docente, 23 de noviembre de 2017).

En el caso en estudio, en primera instancia, se puede observar cómo en la mayoría de las ocasiones la distribución del estudiantado estuvo orientada a la persona docente. En segunda instancia, se evidenció que el tipo de comunicación que prevalece entre docente y el estudiantado fue unidireccional. En cambio, entre el alumnado el tipo de comunicación desarrollada si fue bidireccional, lo que influyó en que en ciertos espacios 
del aula los niveles de intensidad de la interacción fuesen diferentes y focalizados, esto como tercera instancia. En cuarta instancia, la movilidad fue monopolizada por el docente y el rol que ejerce, dado que la distribución en el espacio limitó la capacidad de movilización del estudiantado.

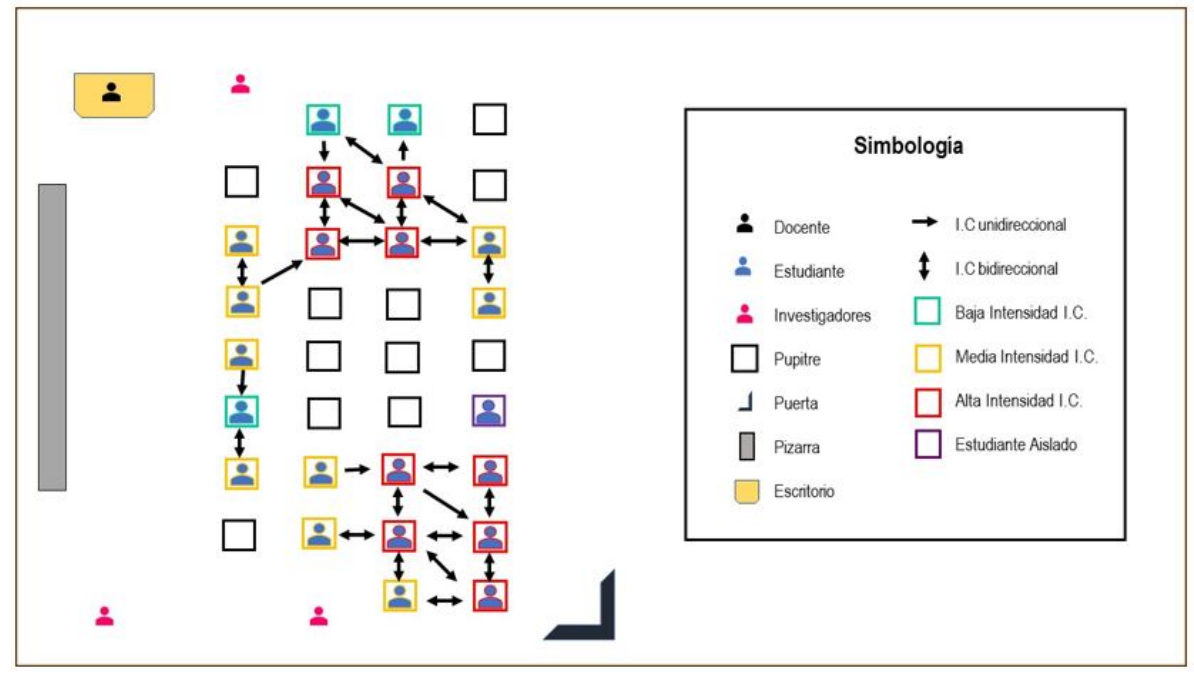

FIGURA 2.

Mapa de las redes de interacción comunicativa

Fuente: Barrientos et al. (2018)

Acorde con lo señalado por Albadalejo (2007) respecto a los modelos de aula en los centros educativos, la organización del mobiliario le Brinda a la persona docente más espacio para desplazarse, mientras que en el caso del estudiantado se reduce debido a la necesidad de permanecer en su asiento de forma duradera.

\subsection{La mediación didáctica docente y la interacción con los contenidos de Estudios Sociales}

En esta categoría de análisis se desarrollaron los siguientes indicadores: temas de estudio de la asignatura, la metodología y actividades, así como de la percepción de las partes involucradas en el proceso educativo.

El presente apartado, tiene relación con la naturaleza de la asignatura de Estudios Sociales, que se enmarca en el estudio de fenómenos que ocurren en la sociedad. Al considerarse, como se indicó previamente, que las interacciones están vinculadas a un contexto social, las temáticas permiten nutrirse y promover la interacción social en las que se engloban las interacciones comunicativas en la clase.

En relación con la asignatura, el tema de estudio al momento del desarrollo de la investigación fue: Dinámica y movilidad espacial: Una mirada a problemáticas contemporáneas. En ella, a pesar de que la persona docente manifestó que era la temática que, a su consideración, era más accesible para el estudiantado por estar relacionada con su realidad, en la mayoría de las clases observadas la interacción desarrollada fue principalmente con el libro de texto. No obstante, cuando los contenidos fueron mediados de una forma que permitiera el acercamiento a la cotidianidad, se promovió una mayor intensidad en las interacciones comunicativas.

El estudiantado, durante un grupo de discusión, reconoce lo anterior al mencionar que:

A mí me gusta más que... no me gusta tantos vídeos, porque es muy... sí, nos da sueño... muy largos... Toda la lección, duran todas las 2 lecciones... también, digamos..., también que nos ponga una práctica o algo así; porque también a veces nos pone a decir lo que opinamos del vídeo, también como que haga algo para que pongamos atención o algo así no sé; si nos pone solo el vídeo nosotros no vamos hacer nada o no vamos a poner atención. (Grupo de discusión 3, 25 de setiembre de 2017) 
Por ende, la interacción que pueden desarrollar los temas debe partir de la cotidianidad. Sobre esto, Liceras y Romero (2016) plantean que las ciencias sociales tienen que estar referidas al medio en que viven y se desenvuelven; Pagés (2009) expone que el alejamiento de las problemáticas sociales tiene influencia en la formación del estudiantado. Por esta razón, el personal docente debe equilibrar la vinculación entre el estudiantado y el contenido, donde cada docente intermedia entre el alumnado, su contexto y los contenidos de la asignatura de Estudios Sociales; dado que, como expresa el mismo estudiantado, prefieren aprender de la persona docente que solo del libro de texto. Aquí es fundamental que los temas no se vean de forma abstracta, para que permita desarrollar la interacción comunicativa entre estudiantes, contenido y contexto.

En relación con la percepción de los actores educativos en torno a las temáticas de la asignatura, se vinculan con las experiencias, expectativas y la realidad. Según el criterio expuesto por la persona docente, hay contenidos cuya naturaleza permite un abordaje más interactivo con el estudiantado, mientras que hay otros que, al ser poco cercanos a su cotidianidad, dificultan propiciar una interacción activa. Ahora bien, en este punto es importante tener presente que la percepción que tenga el personal docente de los temas puede preconcebir la mediación y el manejo de las temáticas en la clase. Por ejemplo, la persona docente entrevistada reconoce que su mediación didáctica es magistral y, por tanto, podría limitar las interacciones comunicativas que se desarrollan en la clase.

De acuerdo con las tradiciones epistemológicas, según el trabajo de campo, el personal docente se encuentra enmarcado en la tradición positivista, debido al rol pasivo que asigna a sus estudiantes y al predominio de clases magistrales; sin embargo, por el trato amigable que la mayoría del estudiantado menciona en los grupos de discusión anteriormente referidos, presenta características de una tradición humanista, tal y como señalan Benejam y Pagés (1997), puesto que establece una relación de empatía con el alumnado. Cabe aclarar, que esto no engloba al personal docente dentro de la tradición humanista.

En relación con lo anterior, Liceras (2016) plantea que “...los docentes no sólo transmiten conocimientos, sino que también contribuyen al progreso adecuado o al retraso en el desarrollo del alumno o alumna como persona, y en sus aspectos psicológicos y sociales" (p. 99). Por lo tanto, que el personal docente sea una figura cercana y amable, podría generar motivación en el estudiantado hacia la asignatura dado el ambiente que en ella se genera.

Por esto, la motivación del personal docente hacia los contenidos de la asignatura es trascendental, ya que influye directamente en la percepción que pueda generar en el estudiantado, que señalan que la asignatura y la persona docente lo motiva a asistir a la clase, pero que en otras ocasiones la mediación que realiza el profesorado no le genera interés hacia los temas por la forma en que se utilizan los recursos didácticos.

\subsection{Empatías o distanciamientos de las interacciones comunicativas en el espacio de clase}

Esta categoría constituye una aproximación teórica construida a partir del trabajo de campo, así como de los hallazgos de la investigación. A continuación, en la categoría central:

...se definen las empatías o distanciamientos de las interacciones comunicativas en el espacio de clase como: un proceso comunicativo que integra distintos tipos de lenguaje, que propician relaciones sociales y académicas influenciadas por un contexto, en un espacio dinámico de aula, en el cual se median contenidos disciplinares en la búsqueda del desarrollo de los procesos de enseñanza y aprendizaje. (Barrientos et al., 2018, p. 180)

Por lo tanto, a partir de esta consideración, se debe comprender que el hecho educativo es complejo y va más allá de lo que la formación académica tradicionalmente ha ofrecido en la formación docente, dado que en la práctica existen más elementos a considerar en los procesos de enseñanza y aprendizaje; para este caso, todo lo que engloba la dinámica de las interacciones comunicativas en educación. 


\section{Conclusiones}

Las interacciones comunicativas que se desarrollan en la clase de Estudios Sociales influyen en los procesos de enseñanza y aprendizaje dadas las evidencias planteadas en las categorías de análisis que convergen en la categoría central, al mostrar que las interacciones comunicativas en educación se configuran de diversos factores, y que la influencia de estos factores dependerá del contexto específico en análisis.

En relación con los elementos comunicativos de la clase, estos constituyen la base de las interacciones comunicativas que se desarrollan, lo que posibilita construir códigos que permiten el entendimiento mutuo entre participantes del proceso educativo; por lo tanto, con el fin de romper esquemas tradicionales unidireccionales, se deben considerar modelos comunicativos bidireccionales, que fomenten una mayor participación del estudiantado, y que no sea exclusividad del personal docente.

En cuanto a las relaciones académico-personales en la clase, estas deben ser horizontales para que se permita el desarrollo de interacciones comunicativas bidireccionales, pero en el entendido de que la persona docente no es igual al estudiantado por el rol que ejerce y desempeña en el aula. Cabe agregar que el contexto sociocultural será determinante en la forma en cómo se desarrollen dichas relaciones.

En lo referente al espacio, movilidad y organización del aula, se evidencia que estos elementos configuran el entramado de redes y radios de interacción comunicativa, por lo tanto, según la forma en que se interrelacionen u organicen, repercuten directamente en los procesos de enseñanza y aprendizaje. Además, se logró observar que estos aspectos tienen más relevancia en el hecho educativo del que tradicionalmente se percibe.

Con respecto a la mediación didáctica del personal docente y la interacción con los contenidos de Estudios Sociales, se concluye que, para que influyan en las interacciones comunicativas y los procesos de enseñanza y aprendizaje, los conocimientos deben partir de la cotidianidad a la que está adscrito el estudiantado; dada la naturaleza de la asignatura al estudiar temáticas sociales, estas permiten ser más próximas a dicha realidad. Si el estudiantado está familiarizado con el conocimiento o la información, más posibilidades existen de generar una interacción estudiante-docente-contenido-contexto.

Por consiguiente, es necesario destacar que cada uno de los apartados analizados, debe ser de conocimiento del cuerpo docente y planificados en su práctica profesional, para que desde la mediación didáctica que se realice, pueda potenciar las interacciones comunicativas en los procesos de enseñanza y aprendizaje.

Por otro lado, se destaca la importancia del método fenomenológico utilizado en la investigación, dado que permitió estudiar una realidad concreta por medio de la experiencia y la percepción de las partes involucradas en los procesos educativos, a partir de un proceso de reflexión, análisis y reinterpretación del objeto de estudio. El método constituyó una guía para responder a las interrogantes de la investigación con la aplicación de las técnicas investigativas, las cuales permitieron estudiar la cotidianidad de la clase de Estudios Sociales y la vivencia de quienes participan en ella.

Por último, dado que este estudio tiene énfasis en la asignatura de Estudios Sociales, se recomienda realizar estudios en otras asignaturas con el fin de investigar si existen puntos en común y si varían las perspectivas de análisis. Asimismo, se deben considerar los demás niveles educativos de secundaria, con el fin de indagar si los alcances de la investigación son similares o distintos en otras realidades educativas.

\section{ReFERENCIAS}

Aguado, J. (2004). Introducción a las teorias de la comunicación y la información. Universidad de Murcia.

Albadalejo, M. (2007). La comunicación más allá de las palabras. Quécomunicamos cuando creemos que no comunicamos. Editorial Graó. 
Alesina, L., Bertoni, M., Mascheroni, P., Moreira, N., Picasso, F., Ramírez, J. y Rojo, V. (1999). Metodología de la investigación en Ciencias Sociales: Apuntes para un curso inicial. Comisión Sectorial de Enseñanza de la Universidad de la República.

Artavia, J. (2005). Interacciones personales entre docentes y estudiantes en el proceso de enseñanza y aprendizaje. Revista Electrónica Actualidades Investigativas en Educación, 5(2), 1-19. http://revistas.ucr.ac.cr/index.php/aie /article/view/9142

Barrientos, A., Córdoba, C. y García, K. (2018). La clase de Estudios Sociales: Un espacio de interacciones comunicativas en los procesos de enseñanza y aprendizaje [Tesis de licenciatura, Universidad de Costa Rica]. http://repositorio .sibdi.ucr.ac.cr:8080/jspui/handle/123456789/7182

Bedmar, S. (2009). La importancia del contexto en el proceso de enseñanza - aprendizaje. Revista Digital para profesionales de la enseñanza, (5), 1-7. https://www.feandalucia.ccoo.es/docuipdf.aspx?d=6448\&s=

Benejam, P. y Pagés, J. (1997). Enseñar y aprender ciencias sociales, geografía e historia en la Educación Secundaria. Editorial Horsori.

Bernstein, B. (1988). Clases, código y control. Hacia una teoría de las transmisiones educativas. Editorial Akal.

Bixio, C. (2002). Enseñar a aprender. Editorial Homo Sapiens.

Bravo, G. y Cáceres, M. (2006). El proceso de enseñanza-aprendizaje desde una perspectiva comunicativa. Revista Iberoamericana de Educación, 38(7), 1-7.

Camacaro de Suárez, Z. (2008). La interacción verbal alumno - docente en el aula de clase (un estudio de caso). Revista Laurus, 14(26), 189-206. http://www.redalyc.org/articulo.oa?id=76111491009

Campos, N., Campos, E., García, N. y Rojas, M. (1993). Conocimiento, participación y cambio: Espacio en el aula. Editorial Universidad de Costa Rica.

Campos, V. y Raubel, R. (2011). La formación del profesional desde una concepción personalizada del proceso de aprendizaje. Cuaderno de educación y desarrollo, 3(28), 1-6. https://dialnet.unirioja.es/servlet/articulo?codigo= 6372722

Cerda, H. (2001). El proyecto de aula: el aula como un sistema de investigación y construcción de conocimientos. Editorial Magisterio.

Chiara, M. (2014). La relación maestro-alumno y su influencia en el aprendizaje, la actitud y el crecimiento personal del alumno [Tesis de maestría, Universidad Internacional de La Rioja]. https://reunir.unir.net/bitstream/handle/ 123456789/2976/MariaChiara_Conidi.pdf?sequence=1

Coll, C. (1984). Estructura grupal, interacción entre alumnos y aprendizaje escolar. Revista Infancia y Aprendizaje, 27(28), 119-138. https://dialnet.unirioja.es/descarga/articulo/668449.pdf

Coll, C. y Sánchez, E. (2008). Presentación. El análisis de la interacción alumno-profesor: Líneas de investigación. Revista de Educación, 346, 15-32. http://www.revistaeducacion.mepsyd.es/re346/re346_01.pdf

De Longhi, A., Ferreyra, A., Peme, C., Bermúdez, G., Quse, L., Martínez S., Iturralde, C., Campaner, G. (2012). Las interacciones comunicativas en clases de ciencias naturales. Un análisis didáctico a través de circuitos discursivos. Revista Eureka sobre enseñanza y divulgación de las ciencias, 9(2), 178-195. http://rodin.uca.es/xmlui/bitstream /handle/10498/14728/1-235-DeLongui.pdf?sequence $=6$

Dirección de Planificación Institucional. (2016). Colegios diurnos y nocturnos. Dependencia pública, privada y privada subvencionada, según dirección regional y circuito escolar, año 2016. Ministerio de Educación Pública. www.mep.go.cr/indicadores_edu/nomina/COLEGIOS.xlsx

Escobar, M. (2015). Influencia de la interacción alumno-docente en el proceso enseñanza-aprendizaje. Revista de Tecnologia y Sociedad, (8). http://www.udgvirtual.udg.mx/paakat/index.php/paakat/article/view/230/347

Freire, P. (2002). Pedagogía de la esperanza. Un reencuentro con la pedagogía del oprimido. Editorial Siglo XXI.

García, E., García, A. y Reyes, J. (2014). Relación maestro alumno y sus implicaciones en el aprendizaje. Ra Ximhai, 10(5), 279-290. http://www.redalyc.org/pdf/461/46132134019.pdf 
Granja, C. (2013). Caracterización de la comunicación pedagógica en la interacción docente - alumno. Revista Investigación en Enfermeria: Imagen y Desarrollo, 15(2), 63-93. https://www.redalyc.org/pdf/1452/14522980 3005.pdf

Guardia de Viggiano, N. (2009). Lenguaje y comunicación. Colección Pedagógica Formación Inicial de Docentes.

Gurdián, A. (2007). El Paradigma Cualitativo en la Investigación Socio - Educativa. Coordinación Educativa y Cultural Centroamericana (CECC), Agencia Española de Cooperación Internacional (AECI).

Herrero, P. (2012). La interacción comunicativa en el proceso de enseñanza - aprendizaje. Revista electrónica de investigación, 1, 138-143. http://www.ugr.es/ miguelgr/ReiDoCrea-Vol.1-Art.19-Herrero.pdf

Husserl, E. (1949). Ideas relativas a una fenomenologia pura y una filosofía fenomenológica. Fondo de Cultura Económica.

Jaramillo, L. y Aguirre, J. (2012). Aporte del método fenomenológico a la investigación educativa. Revista Latinoamericana de Estudios Educativos, 8(2), 51-74. http://www.redalyc.org/pdf/1341/134129257004.pdf

Jauregi, K. (2012, 19-22 de setiembre). La interacción, elemento clave en el proceso de aprendizaje de ELE. [Conferencia]. XXIII Congreso internacional de la ASELE, Plurilingüismo y enseñanza de ELE en contextos multiculturales. Girona, España.

Juárez, A. (2019). La incidencia del lenguaje no verbal en la interacción didáctica en universidad veracruzana [Tesis doctoral inédita]. Universidad de Xalapa.

Kaplún, M. (2002). Una pedagogía de la comunicación (el comunicador popular). Editorial Caminos.

Keidar, D. (2005). La comunicación en el aula: Uso de la inteligencia emocional y la comunicación no verbal en la enseñanza de ética en la escuela de medicina. Universidad de los Andes.

Keil, G. (2011). Comunicación en el ámbito escolar en relación con los procesos de enseñanza y aprendizaje: Análisis de la interacción docente-alumno. [Tesis de licenciatura inédita]. Universidad Abierta Interamericana. http://imgbib lio.vaneduc.edu.ar/fulltext/files/TC104122.pdf

Liceras, A. (2016). Las dificultades en la enseñanza y el aprendizaje de las Ciencias Sociales. En A. Liceras y G. Romero, (Comps.), Didáctica de las Ciencias Sociales (pp. 95-115). Pirámide.

Liceras, A. y Romero, G. (2016). Didáctica de las Ciencias Sociales. Madrid: Pirámide.

McMillan, J. y Schumacher, S. (2005). Investigación educativa. Madrid: Pearson.

Mejía, A. y Ávila, L. (2009). Relaciones sociales e interacción en el aula en secundaria. Revista Mexicana de Investigación Educativa, 4(14), 485-513. http://www.redalyc.org/articulo.oa?id=14004107

Meneses, G. (2007). El proceso de enseñanza-aprendizaje: El acto didáctico. Revista de medios y educación, (29), 31-65.

Ministerio de Educación Pública [MEP]. (2016). Programa de Estudios de la asignatura de Estudios Sociales para III Ciclo de la Educación General Básica y la Educación Diversificada. Imprenta Nacional.

Moreno, J. (2007). Habilidades sociales e intervención docente. En Á. Sanz (Ed.), Laeducación lingüistica y literatura en secundaria, (pp. 307-320). Dirección General de Formación Profesional e Innovación Educativa.

Motta, C. (2010). Relación centro educativo, familia y comunidad: Estrategia clave para mejorar la calidad de la educación. EUNED.

Moya, A. (2010). La relación profesor - alumno. Revista digital innovaciones y experiencias educativas, (27), 1-9. https://archivos.csif.es/archivos/andalucia/ensenanza/revistas/csicsif/revista/pdf/Numero_27/ANTON IA_MARIA_MOYA_MARTINEZ.pdf

Pacheco, L. (2013). Clima escolar: Percibido por alumnos (as) y profesores (as) a partir de las relaciones sociales que predominan en las aulas de la clase del instituto polivalente Dr. Doroteo Varela Mejía de Yarumela La Paz [Tesis de maestría, Universidad Pedagógica Nacional Francisco Morazán]. http://www.cervantesvirtual.com/nd/ark :/59851/bmchh8f3

Pagés, J. (2009, 26-28 de agosto). Enseñar y aprender ciencias sociales en el siglo XXI: Reflexiones casi al final de una década. [Conferencia]. II Congreso Internacional Investigación en Educación y Formación Docente. Medellín, Colombia.

Programa del Estado de la Nación. (2015). Quinto Informe del Estado de la Educación. Edigital ED. 
Keiner García-CaLderón, et aL. Las interacciones comunicativas en los procesos de enseñanZa y aPre...

Programa del Estado de la Nación. (2017). Sexto Informe del Estado de la Educación. Servicios Gráficos, A. C.

Rizo, M. (2007). Interacción y comunicación en entornos educativos: Reflexiones teóricas, conceptuales y metodológicas. Revista da Associação Nacional dos Programas de Pós-Graduação em Comunicação, 8, 1-16. http ://dx.doi.org/10.30962/ec.v8i0.143

Sandoval, M. (2014). Convivencia y clima escolar: Claves de la gestión del conocimiento. Revista Última Década, 22(41), 153-178. http://www.scielo.cl/scielo.php?pid=S0718-22362014000200007\&script=sci_arttext

Sime, L. (2006). Las relaciones interpersonales en la educación desde el paradigma de la convivencia. Revista Educación, 16(30), 41-52. http://revistas.pucp.edu.pe/index.php/educacion/article/viewFile/1800/1741

Torres, J. (1998). El curriculum oculto. Editorial Morata.

Vigotsky, L. (1991). Acerca del desarrollo cultural del niño. Universidad de Moscú.

Vigotsky, L. (1995). Pensamiento y lenguaje. Teoria del desarrollo cultural de las funciones psíquicas. Ediciones Fausto.

\section{INFORMACIÓN ADICIONAL}

Cómo citar: Cómo citarGarcía-Calderón, K., Barrientos-Córdoba, A. E. y Córdoba-Alfaro, C. I. (2022). Las interacciones comunicativas en los procesos de enseñanza y aprendizaje en la clase de Estudios Sociales. Revista Educación, 46(1). http://doi.org/10.15517/revedu.v46i1.47660 